\title{
Spatial Resolution Attainable in Germanium Detectors by Pulse Shape Analysis
}

\author{
J. Blair \\ Bechtel, Nevada \\ North Las Vegas, NV 89130 \\ and \\ D. Beckedahl, J. Kammeraad, G. Schmid \\ Lawrence Livermore National Laboratory \\ Livermore, CA 94550
}

This paper was prepared for submittal to the

1998 Symposium on

Radiation Measurements and Applications

Ann Arbor, MI

May 12-14, 1998

May, 1998

This is a preprint of a paper intended for publication in a journal or proceedings. Since changes may be made before publication, this preprint is made available with the understanding that it will not be cited or reproduced without the permission of the author. 


\section{DISCLAIMER}

This document was prepared as an account of work sponsored by an agency of the United States Government. Neither the United States Government nor the University of California nor any of their employees, makes any warranty, express or implied, or assumes any legal liability or responsibility for the accuracy, completeness, or usefulness of any information, apparatus, product, or process disclosed, or represents that its use would not infringe privately owned rights. Reference herein to any specific commercial product, process, or service by trade name, trademark, manufacturer, or otherwise, does not necessarily constitute or imply its endorsement, recommendation, or favoring by the United States Government or the University of California. The views and opinions of authors expressed herein do not necessarily state or reflect those of the United States Government or the University of California, and shall not be used for advertising or product endorsement purposes. 


\title{
SPATIAL RESOLUTION ATTAINABLE IN GERMANIUM DETECTORS BY PULSE SHAPE ANALYSIS
}

J. Blair, Bechtel Nevada, North Las Vegas, NV 89130, D. Beckedahl, J. Kammeraad and G. Schmid, Lawrence Livermore National Laboratory, Livermore, CA 94550

This work was supported by the U.S. Department of Energy, Nevada Operations Office, under Contract No. DE-AC08-96NV11718 and by the U.S. D.O.E. under contract W-7405-Eng-48 by Lawrence LivemoreNational Laboratory.

\begin{abstract}
There are several applications for which it is desirable to calculate the locations and energies of individual gamma-ray interactions within a high purity germanium (HPGe) detector. These include gamma-ray imaging and Compton suppression. With a segmented detector this can be accomplished by analyzing the pulse shapes of the signals from the various segments. We examine the fundamental limits to the spatial resolution attainable with this approach. The primary source of error is the series noise of the field effect transistors (FETs) at the inputs of the charge amplifiers. We show how to calculate the noise spectral density at the output of the charge amplifiers due to an optimally selected FET. This calculation is based only on the detector capacitance and a noise constant for the FET technology. We show how to use this spectral density to calculate the uncertainties in parameters, such as interaction locations and energies, that are derived from pulse shape analysis using maximum likelihood estimation (MLE) applied to filtered and digitized recordings of the charge signals. Example calculations are given to illustrate our approach. Experimental results are given that demonstrate that one can construct complete systems, from detector through data analysis, that come near the theoretical limits.
\end{abstract}




\section{Introduction}

There are applications in which one must determine the positions of individual gamma-ray interactions with an HPGe detector $[1,2]$. To determine all three coordinates of interactions requires a segmented detector with possibly many signal processing channels. It is desirable to calculate the limits of the resolution of a particular detector configuration without the expense of building a system and carrying out complex experiments. We present here a procedure for carrying out such calculations. It is based on the observation that the dominant error source is the noise from the field effect transistor (FET )at the input of the detector preamp. We model the attainable noise vs. input capacitance for FETs, calculate the noise output for an optimally selected FET and use this result to estimate the errors in a maximum likelihood estimate of the interaction locations.

\section{FET noise}

Figure 1 depicts a detector (segment) connected to the input stage of a charge amplifier. The voltage signal, $v_{n}$, is the FET series noise voltage. It is not an actual voltage source, but is the voltage at the gate of the FET that would be required to generate the thermal noise current in the channel of the FET. The noise voltage, $v_{n}$, is well known $[3,4]$ to be related to the mutual conductance, $g_{m}$, of the FET by the relation

$$
v_{n}^{2} \cong \frac{4 k T B \times .7}{g_{m}},
$$

where $k$ is the Boltzman constant, $T$ is the absolute temperature, and $B$ is the bandwidth over which the noise measurement is made. From this relation we can see that the noise can be made as small as desired by increasing $g_{m}$; however, increasing $g_{m}$ increases the input capacitance, $C_{F}$, which decreases the signal. 
An important figure of merit for FETs used in charge amplifiers is the ratio, $g_{m} / C_{F}$. This gives the "charge sensitivity" of the FET and is limited by how short the channel can be made [4] by the relation

$$
\frac{g_{m}}{C_{F}}=\frac{8}{9} \frac{\mu W_{00}}{L^{2}},
$$

where $\mu$ is the majority carrier mobility, $W_{00}$ is the pinch-off voltage, and $L$ is the length of the channel controlled by the gate. Combining (1) and (2) we have

$$
v_{n}^{2} C_{F} \cong \frac{3 k T L^{2} B}{\mu W_{00}}=K B,
$$

where $K$ is a constant which depends on the parameters in (2). Note that although $K$ is proportional to the absolute temperature, $T$, it is the temperature of the majority carriers in the FET channel that is relevant. Even if the exterior of the FET is cooled to liquid nitrogen temperature, the channel is in electrical contact, and hence very good thermal contact, with preamplifier components at room temperature, and, hence the cooling has little effect on the noise. However, placing the FET near the detector in the liquid nitrogen effects the signal to noise ratio by decreasing the parasitic capacitance of the leads between the detector and the FET.

The constant, $K$, in (3) is a measure of the state-of-the-art in low noise FET manufacturing. Varying the width of the channel, keeping other design parameters constant, changes $C_{F}$ and $v_{n}$ in a manner that keeps $K$ constant. Placing an ideal transformer at the input of the transistor changes the effective $C_{F}$ and $v_{n}$ at the input of the transformer in a manner that leaves $K$ invariant. Circuits made of several transistors in either parallel (increases capacitance and gain, decreases noise) or in Darlington combination (decreases capacitance and gain, increases noise) also leave $K$ invariant. 
If $v_{n}$ is expressed in $\mathrm{nV} / \sqrt{\mathrm{Hz}}$ and $C_{F}$ is expressed in $\mathrm{pF}$, the value, $\mathrm{K}=4$ represents current technology. In $[4,1973]$ an experimental device, SFC6593, was reported with $K=$ 4.7. The two commercial devices reported in the same source had $K$ values of 8 and 16.

In [5, 1992] devices with $C_{F}=1$ and $K=4$ and with $C_{F}=8$ and $K=3.4$ were reported. These devices were not optimized solely for sensitivity and noise performance [6], so devices with smaller $K$ may be possible. The measured value for our experimental system [1 is $K=10$. This was obtained by measuring the total noise at the output of the amplifier system and attributing it all to FET noise.

\section{Optimum noise level}

We desire to evaluate detector performance for the situation in which the detector is mated with the best possible buildable FET. The signal voltage due to charge $Q$ at the input of the FET is $Q /\left(C_{D}+C_{F}\right)$, where $C_{D}$ is the detector capacitance. The noise per unit bandwidth, from (3), is $v_{n}=\sqrt{K / C_{F}}$. The maximum of the ratio of these two quantities occurs at

$$
C_{F}=C_{D}
$$

The relationship (4) only holds if one assumes that $v_{n}^{2} C_{F}=$ const. If you minimize with another relation, such as $v_{n}^{p} C_{F}=$ const., with $\mathrm{p} \neq 2$, the relationship between $C_{D}$ and the optimum $C_{F}$ will be different. The noise voltage per unit bandwidth from (3) is then

$$
e_{n}=\sqrt{K / C_{D}}
$$

This can be converted into energy units by multiplying by $10^{-9}$ times the total capacitance, $2 C_{D}$, to convert from $\mathrm{nV}$ to $\mathrm{pC}$, then dividing by the $.053 \mathrm{pC} / \mathrm{Mev}$ sensitivity of $\mathrm{Ge}$. This gives 


$$
E_{n}=3.8 \times 10^{-8} \sqrt{K C_{D}}=7.6 \times 10^{-8} \sqrt{C_{D}} \mathrm{Mev} / \sqrt{\mathrm{Hz}}
$$

where $C_{D}$ is in pC. The second equality assumes that $K=4$.

Figure 2 shows the assumed mechanism for measuring the charge signal from each detector segment. The amplifier is assumed to have a flat frequency response up to the Nyquist frequency of the waveform digitizer. The waveform digitizer samples with a time spacing of $\Delta t$. The anti-aliasing filter is a low-pass filter with a bandwidth of $1 /(2 \Delta t)$. We assume that the sampled data is scaled to be in units of Mev and that the only source of error is the noise from the FET.

Substituting the bandwidth of $1 /(2 \Delta t)$ into (6) gives

$$
\sigma=2.7 \times 10^{-8} \sqrt{C_{D} / \Delta t} \mathrm{Mev}
$$

for the standard deviation of any sampled value of the digitized signal. The units for $C_{D}$ and $\Delta t$ are $\mathrm{pC}$ and Seconds. Because the noise is due to thermal currents, the errors have a Gaussian distribution with zero mean [7, p. 189]. Because the noise is white, the errors in different sample values are independent [7, p. 145-155]. Thus, if $\boldsymbol{e}=\left(e_{1}, e_{2}, \ldots, e_{\mathrm{m}}\right)^{\mathrm{T}}$ is a column vector of errors in sampled values, the probability density is given by

$$
P\left(e_{1}, e_{2}, \ldots e_{m}\right)=\prod_{k=1}^{m} \frac{1}{\sqrt{2 \pi \sigma^{2}}} \exp \left[-\frac{e_{k}^{2}}{2 \sigma^{2}}\right]=\left(2 \pi \sigma^{2}\right)^{-m / 2} \exp \left[-\frac{\boldsymbol{e}^{T} \boldsymbol{e}}{2 \sigma^{2}}\right]
$$

It may appear from (7) that making $\Delta t$ too small could be deleterious, because the standard deviation of the error increases as $\Delta t$ decreases. However, we will make clear later why this is not the case. 
In practice, the recorded signals will have a baseline offset due to accumulated charge from previous pulses and due to excess low frequency noise that isn't accounted for in (1). The analysis given here assumes that this error has been corrected.

\section{Estimating parameters and their errors}

The data by which we determine interaction locations are the recorded signals, through a system such as that in Figure 2, from each of the segments. Recorded signals from each segment have a length of $L$ samples. The number of segments is $N_{S e g}$. Signals are formed into column vectors, $v$, of length $M=L N_{S e g}$ by concatenating the signals from the various segments.

Let $U_{j}(t, \boldsymbol{r})$ be the signal at the $j$ th segment resulting from a single interaction occurring a $t$ $=0$ of $1 \mathrm{Mev}$ energy at (vector) position $\boldsymbol{r}$. In our analysis we assume that the number, $I$, of interactions is known. In practice, one would have to determine this from the data; we won't be covering this aspect of the problem in this paper. The signal from the $j$ th segment resulting from the $I$ interactions is given by

$$
V_{j}(t)=\sum_{i=1}^{I} E_{i} U_{j}\left(t-t_{0}, \boldsymbol{r}_{i}\right)
$$

where $t_{0}$ is the time of the interactions, $E_{i}$ is the energy of the $i$ th interaction, and $\boldsymbol{r}_{\mathrm{i}}$ is the position vector of the $i$ th interaction. Thus, there are $4 I+1$ parameters that must be determined from the data. We assume that the digitized record starts at a $t$ sufficiently earlier than $t_{0}$ that an adequate baseline correction can be made.

Let $\boldsymbol{p}$ denote a column vector consisting of the $4 I+1$ unknown parameters; let $\boldsymbol{v}(\boldsymbol{p})$ be the column vector of the concatenated segment signals that would result from noiseless 
recording of a signal with parameters $\boldsymbol{p}$, and let $\boldsymbol{v}$ be the actual recorded signal. From (8) the likelihood function [8] is given by

$$
L(\boldsymbol{p}, \boldsymbol{v})=\left(2 \pi \sigma^{2}\right)^{-M / 2} \exp \left[-\frac{(\boldsymbol{v}-\boldsymbol{v}(\boldsymbol{p}))^{T}(\boldsymbol{v}-\boldsymbol{v}(\boldsymbol{p}))}{2 \sigma^{2}}\right]
$$

We estimate the unknown parameter $\boldsymbol{p}$ with the vector $\hat{\boldsymbol{p}}$ that maximizes (10). This is equivalent to minimizing $(\boldsymbol{v}-\boldsymbol{v}(\boldsymbol{p}))^{T}(\boldsymbol{v}-\boldsymbol{v}(\boldsymbol{p}))$, a non-linear least squares problem with several known methods of solution [9, p. 681-688]. Our purpose here is to determine the accuracy with which the estimated parameter values approximate the true parameter values. This is an easier problem than that of finding the solution to the least squares problem.

Let

$$
\boldsymbol{S}=\frac{\partial v}{\partial \boldsymbol{p}} \text {, i.e. } S_{r s}=\frac{\partial v_{r}}{\partial p_{s}} .
$$

The condition for a least squares solution is [9, Sec. 14.4]]

$$
S^{T}(\hat{\boldsymbol{p}})(\boldsymbol{v}-\boldsymbol{v}(\hat{\boldsymbol{p}}))=0 .
$$

We let $\boldsymbol{p}_{\mathrm{O}}$ be the true parameter vector and assume that the errors in the parameters are small enough that the first order approximation

$$
v(\hat{\boldsymbol{p}}) \cong v\left(p_{0}\right)+S(\hat{\boldsymbol{p}})\left(\hat{\boldsymbol{p}}-\boldsymbol{p}_{0}\right) .
$$

Since $\boldsymbol{S}$ and $\boldsymbol{S}^{\boldsymbol{T}}$ are always evaluated at $\hat{\boldsymbol{p}}$, we will omit the argument when writing them. Multiplying (13) by $\boldsymbol{S}^{T}$ we obtain 


$$
S^{T} S\left(\hat{p}-p_{0}\right) \cong S^{T}\left(v(\hat{p})-v\left(p_{0}\right)\right)
$$

We can rewrite $\boldsymbol{v}(\hat{\boldsymbol{p}})-\boldsymbol{v}\left(\boldsymbol{p}_{\boldsymbol{0}}\right)$ as $\boldsymbol{v}(\hat{\boldsymbol{p}})-\boldsymbol{v}+\boldsymbol{v}-\boldsymbol{v}\left(\boldsymbol{p}_{\boldsymbol{0}}\right)$ then, using (12) and the fact that $\boldsymbol{v}-\boldsymbol{v}\left(\boldsymbol{p}_{0}\right)=\boldsymbol{e},(14)$ becomes

$$
\boldsymbol{S}^{T} \boldsymbol{S}\left(\hat{\boldsymbol{p}}-\boldsymbol{p}_{0}\right) \cong \boldsymbol{S}^{T} e, \text { or } \Delta \boldsymbol{p}=\left(\hat{\boldsymbol{p}}-\boldsymbol{p}_{0}\right) \cong\left(\boldsymbol{S}^{T} \boldsymbol{S}\right)^{-1} \boldsymbol{S}^{T} e .
$$

This gives the error in the parameters in terms of the errors in the data. Using the fact, from (8), that $\mathrm{E}\left[\boldsymbol{e} \boldsymbol{e}^{\mathrm{T}}\right]=\boldsymbol{\sigma}^{2} \boldsymbol{I}$, where $\boldsymbol{I}$ is the identity matrix, we obtain the covariance matrix, $C$, for $\Delta p$ as

$$
\boldsymbol{C}=\mathrm{E}\left[\Delta p \Delta p^{T}\right]=\left(\boldsymbol{S}^{T} \boldsymbol{S}\right)^{-1} \boldsymbol{S}^{T} \mathrm{E}\left[e e^{T}\right] S\left(S^{T} S\right)^{-1}=\sigma^{2}\left(S^{T} S\right)^{-1},
$$

where $\mathrm{E}[]$ is the statistical expectation operator. The standard deviation of the $i$ th parameter is

$$
\sigma_{i}=\sqrt{C_{i i}} .
$$

Now, $\sigma$ is given by (7), making $\sigma^{2}$ proportional to $1 / \Delta t$. If $\Delta t$ is small enough the sampled signal adequately represents the continuous time signal then

$$
\left(\boldsymbol{S}^{T} \boldsymbol{S}\right)_{r s} \cong \sum_{j=1}^{N_{\text {seg }}} \frac{1}{\Delta t} \int \frac{\partial V_{j}(t)}{\partial p_{r}} \frac{\partial V_{j}(t)}{\partial p_{s}} d t
$$

where $V_{j}(t)$ is given by (9). This implies that $\left(\mathrm{S}^{\mathrm{T}} \mathrm{S}\right)^{-1}$ is proportional to $\Delta t$. Combining this which the $1 / \Delta t$ dependence of $\sigma^{2}$ in (7) means that the covariance matrix in (16) is (for sufficiently small $\Delta t$ ) independent of $\Delta t$. If $\Delta t$ is too large, the anti-aliasing filter in Figure 2 washes out some of the detail in the signals, which decreases the resolution. We 
evaluated the covariance matrix as a function of $\Delta t$ for our $5 \mathrm{~cm}$ by $5 \mathrm{~cm}$ detector and found that it was constant for $\Delta t<20$ nanoseconds ( $25 \mathrm{MHz}$ bandwidth).

\section{Experimental results and example}

Figure 3 shows the noise measured on our experimental system. Shown is the integral of the power spectral density. The slope of the straight line is that given by the square of (6) with $C_{D}=25 \mathrm{pF}$ and $K=10$. The noise spectrum was calculated from a record of data from the waveform digitizer in the configuration shown in Figure 2.

We use the unsegmented cylindrical detector of [1] for an example. We assume a single interaction of .2 Mev and calculate the radial position uncertainty. The three columns of $\boldsymbol{S}$ are given by sampled values of (see (9) and (11))

$$
\frac{\partial V}{\partial t_{0}}=-E \frac{\partial U\left(t-t_{0}, r\right)}{\partial t}, \frac{\partial V}{\partial E}=U\left(t-t_{0}, r\right) \text { and } \frac{\partial V}{\partial r}=E \frac{\partial U\left(t-t_{0}, r\right)}{\partial r}
$$

The function, $U$, was calculated, to 300 nanoseconds after the interaction, by (12-27) of [10] using empirically determined constant electron and hole velocities. The derivative of $U$ with respect to $r$ was approximated using a finite difference with $2 \mathrm{~mm}$ separation. Standard deviations for radial position and energy were calculated as a function of radial position of the interaction using (17), (16) and (9) with $C_{D}=25 \mathrm{pF}$. The results are in Figure 4. From (19) it can be shown that the radial resolution is inversely proportional to energy and that the energy resolution is independent of energy. The energy resolution shown is only that due to FET noise; at higher energies the inherent carrier statistics is a larger effect. The carrier statistics do not affect the radial resolution, but at higher energies the size of the electron cloud will have a larger effect than reported here. The point at which the radial uncertainty is worst $(16 \mathrm{~mm})$ is where the electron and hole travel times are equal and the signal rise time is a minimum. The energy resolution is best at this point, because 
the duration of the full amplitude portion of the signal is maximized. The energy resolution improves as the record length increases, but the position resolution does not.

\section{Conclusions}

We have presented a straight forward procedure for calculating the uncertainties due to FET noise in the positions and energies, calculated by the maximum likelihood method, of gamma-ray interactions in a segmented HPGe detector. The calculations are for an optimally selected FET matched to the capacitance of the detector. A single constant was presented to describe the state-of-the-art of FET technology (as it applies to this problem). The uncertainty calculations can be performed using this constant, without the requirement to obtain and measure an FET or preamplifier. 


\section{References}

[1] G. Schmid, et al, this conference.

[2] J. Kurfess, "Compton scatter imaging in Astrophysics", 1997 IEEE Nuclear Science Symposium, CD-ROM Proceedings.

[3] A. van der Ziel, Proc. IRE, Aug. 1962, pp. 1808-1812.

[4] V. Radeka, IEEE Trans. Nucl. Sci., NS20, (11), pp. 182-189.

[5] T. Nashibi, Nucl., Inst. and Meth., A322 (1992), pp. 551-556.

[6] T. Nashibi, IEEE Trans. Nucl. Sci., NS38, (2), pp. 77-82.

[7] W. Davenport and W. Root, An Introduction to the Theory of Random Signals and Noise, IEEE Press, 1987.

[8] C. R. Rao, Linear Statistical Inference and its Applications, John Wiley \& Sons, 1965.

[9] W. Press, S. Teukolsky, W. Vetterling and B. Flannery, Numerical Recipes in C-The Art of Scientific Computing. Cambridge University Press, 1992.

[10] G. Knoll, Radiation Detection and Measurement, 2nd Edition, John Wiley \& Sons, 1989. 


\section{Figure captions}

1. Equivalent input stage for one detector segment showing the detector, its capacitance, the FET, its capacitance and the equivalent noise generator.

2. Block diagram of the system to record the signal from one detector segment.

3. The measured noise power (integrated power spectrum) as a function of frequency for the example system.

4. Calculated standard deviation of the maximum likelihood estimate of the radial coordinate and the energy for the example in the text. 


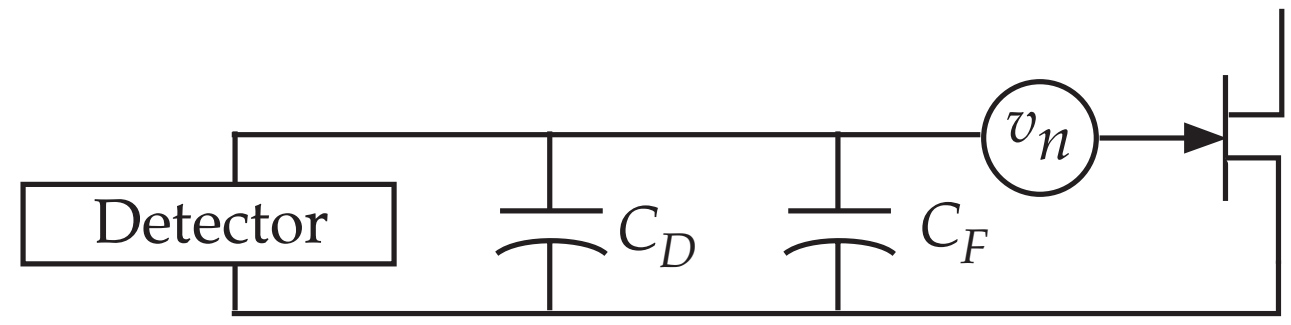




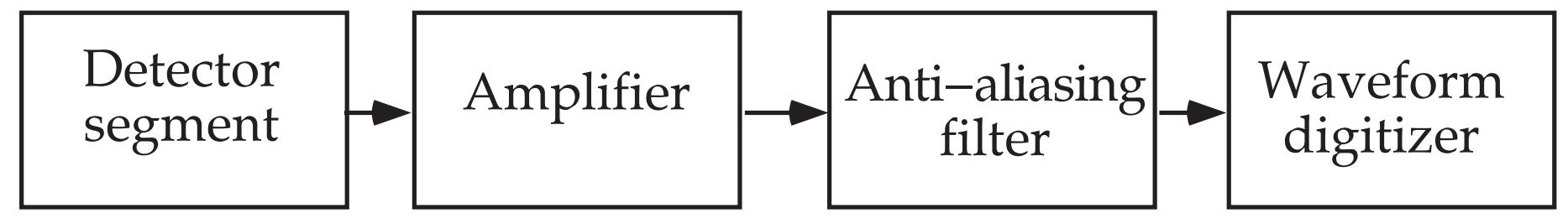




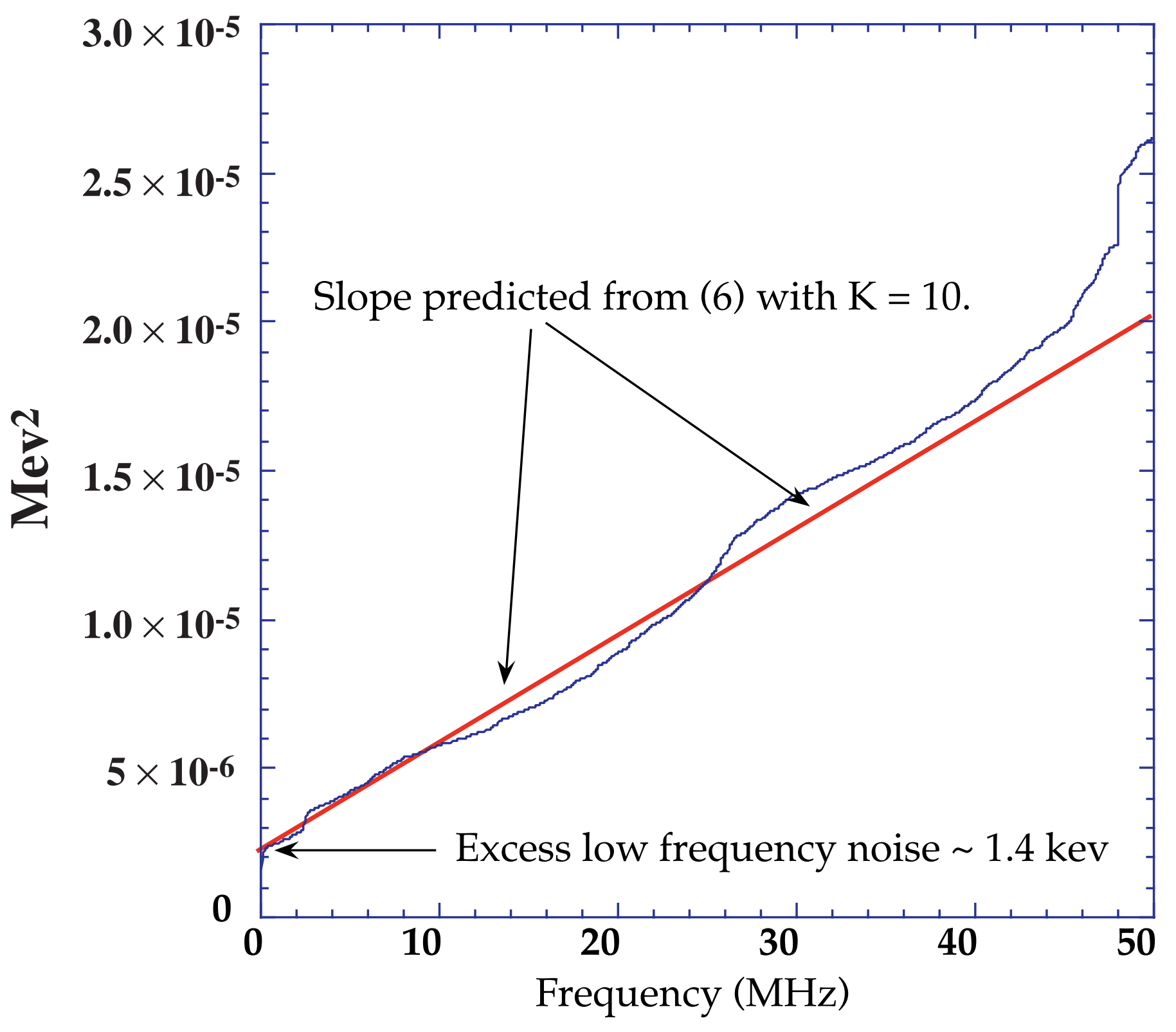




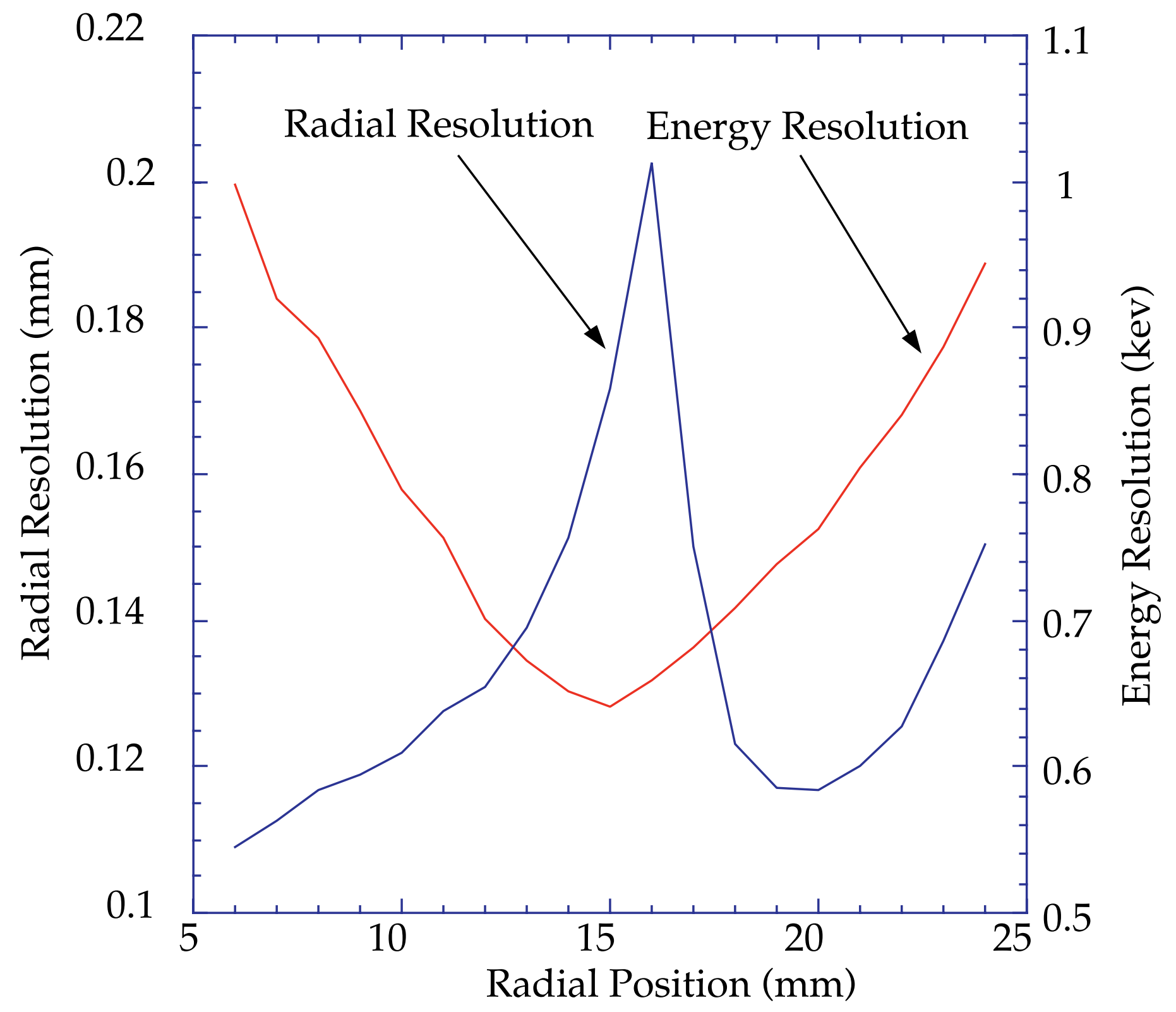




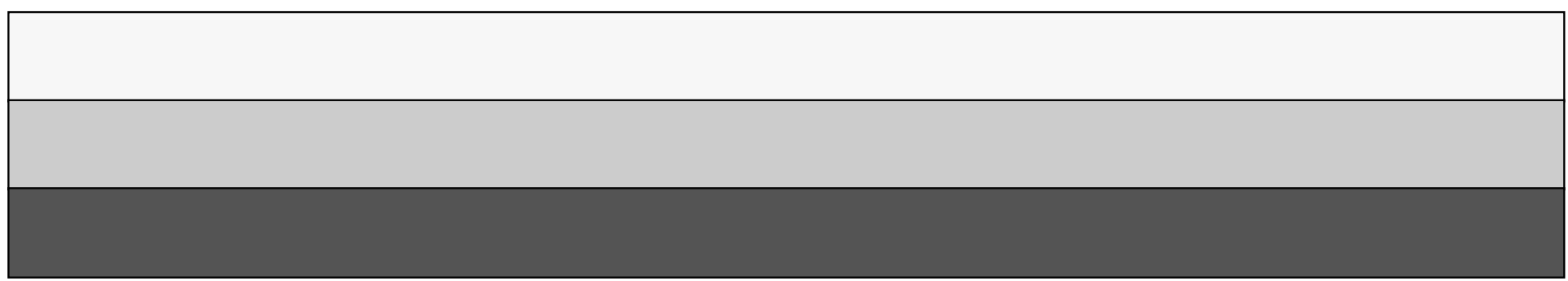

